Tomasz Stachura, ${ }^{1,}$, Natalia Celejewska-Wójcik, ${ }^{1,2}$ Kamil Polok ${ }^{1,3}$, Karolina Górka ${ }^{1,2}$, Sabina Lichołai, ${ }^{4,}$, Krzysztof Wójcik', Jacek Krawczyk ${ }^{1,2}$, Anna Kozłowska', Marek Przybyszowski ${ }^{1,2}$, Tomasz Włoch ${ }^{1,6}$, Jacek Górka ${ }^{3}$, Krzysztof Sładek ${ }^{1,2}$

'Department of Pulmonology and Allergology, University Hospital in Kraków, Kraków, Poland

${ }^{2} 2^{\text {nd }}$ Chair of Internal Medicine, Jagiellonian University Medical College, Kraków, Poland

${ }^{3}$ Department of Intensive Care and Perioperative Medicine, Kraków, Poland

${ }^{4}$ Division of Molecular Biology and Clinical Genetics, Faculty of Medicine, Jagiellonian University Medical College, Kraków, Poland

${ }^{5}$ Sano Centre for Computational Medicine, Kraków, Poland

${ }^{6}$ Department of Rehabilitation in Internal Diseases, Institute of Clinical Rehabilitation, University School of Physical Education, Kraków, Poland

\title{
A clinical profile and factors associated with severity of the disease among Polish patients hospitalized due to COVID-19 an observational study
}

\begin{abstract}
Introduction: The coronavirus disease 2019 (COVID-19) is one of the greatest clinical challenges of the last decades. Clinical factors associated with severity of the disease remain unclear. The aim of the study was to characterize Polish patients hospitalized due to COVID-19 and to evaluate potential prognostic factors of severe course of the disease.

Material and methods: An observational study was conducted from March to July 2020 in the Pulmonology and Allergology Department of the University Hospital in Kraków, Poland. Consecutive patients with confirmed SARS-CoV-2 (Severe Acute Respiratory Syndrome Coronavirus 2) infection were enrolled, and data about past medical history, signs and symptoms, laboratory results, imaging studies results, in-hospital management and outcomes was prospectively gathered.

Results: The study sample comprised 100 patients at the mean age of 59.2 (SD 16.1) years among whom 63 (63.0\%) were male. Among them $10(10.0 \%)$ died, $47(47 \%)$ presented respiratory failure, $15(15.0 \%)$ were transferred to the intensive care unit, $17(17.0 \%)$ developed acute kidney injury, 7 (7.0\%) had sepsis and $10(10.0 \%)$ were diagnosed with pulmonary embolism. Multivariable analysis revealed age (OR 1.1; 95\% $\mathrm{Cl} 1.01-1.15)$, body mass index (BMl; OR 1.24; 95\% Cl 1.01-1.53), modified early warning score (MEWS; OR 3.95; 95\% $\mathrm{Cl} 1.48-12$ ), the highest d-dimer value (OR 1.73; 95\% $\mathrm{Cl} 1.03-2.9$ ) and lactate dehydrogenase (LDH; OR 1.16; $95 \% \mathrm{Cl} 1.03-1.3$ ) to be associated with severe course of COVID-19.

Conclusion: This observational study showed that almost half of hospitalized patients with COVID-19 developed respiratory failure in the course of the disease. Increasing age, BMI, MEWS, d-dimer value and LDH concentration were associated with the severity of COVID-19.
\end{abstract}

Key words: clinical characteristics, coronavirus disease 2019, respiratory failure, risk factors, SARS-CoV-2

Adv Respir Med. 2021; 89: 124-134

\section{Introduction}

Clinical presentation of COVID-19 is highly variable and involves multiple organs, however, the respiratory system seems to be predominantly affected. There are several reports describing an increased incidence of injuries to the heart, kidneys, muscles, gastrointestinal tract and the nervous system among COVID-19 patients [1-3]. Another significant factor influencing outcomes of persons infected with SARS-CoV-2 is an increased incidence of thromboembolic complications which is secondary to severe disturbances in coagulation and fibrinolysis as well as endothelial damage, sometimes referred to as COVID-19-associated coagulopathy $[4,5]$. It is estimated that 
SARS-CoV-2 infection remains mildly symptomatic in about $80 \%$ of patients. However, approximately $15 \%$ of cases are severe, warranting hospitalization and the remaining $5 \%$ of patients are critically ill and require management in the intensive care unit (ICU) [6]. At the time of the study, only systemic glucocorticoids were recognized to reduce mortality in several randomized controlled trials [7].

The objective of this study was to characterize the first COVID-19 patients treated in the Pulmonology and Allergology Department of the University Hospital in Kraków, Poland in terms of patients' clinical, laboratory, diagnostic outcomes and factors associated with the severity of the disease.

\section{Material and methods}

\section{Study design}

This is an observational study conducted from March to July 2020 in the Pulmonology and Allergology Department, University Hospital in Kraków, Poland. The study was approved by the Ethics Committee of Jagiellonian University Medical College, Kraków, Poland (KBET 1072.6120.145.2020), and written informed consent was obtained from all participants.

\section{Study sample and data collection}

We enrolled all consecutive patients with SARS-CoV-2 infection confirmed using reverse transcription polymerase chain reaction, admitted to the Pulmonology and Allergology Department. We collected basic demographic (age, sex) and detailed clinical information about the patients (symptoms, comorbidities, medications, smoking status) based on the interviews and comprehensive analysis of medical records. In each patient, modified early warning score (MEWS) was calculated on admission. We recorded laboratory results on the day of admission, on the $2^{\text {nd }}$ to $4^{\text {th }}$ day of hospitalization and the $6^{\text {th }}$ to $8^{\text {th }}$ day of hospitalization as well as results of imaging studies (chest X-ray and computed tomography of the chest).

\section{Outcomes}

The patients were observed during the entire hospitalization in the University Hospital for the occurrence of the following outcomes: death, myocardial injury, myocardial infarction (both defined according to the Fourth Universal Definition of Myocardial Infarction [8]), stroke (defined as a new focal neurological deficit with signs and symptoms lasting more than 24 hours), acute kidney injury (AKI, diagnosed according to the Kidney Disease Improving Global Outcomes definition) [9], sepsis, septic shock (both defined according to the 2016 Surviving Sepsis Campaign International Guidelines for Management of Sepsis and Septic Shock) [10], pulmonary embolism (diagnosed with computed tomography pulmonary angiogram) as well as transfer to the ICU and a need for mechanical ventilation.

\section{Statistical analysis}

Categorical variables were presented as numbers (percentages), whereas continuous variables were reported as medians (interquartile range) or means (standard deviation) depending on variable distribution. Associations of quantitative data were analyzed with Student $t$ test and with the nonparametric Mann-Whitney U test. To assess the association between selected factors and a severe course of the disease (defined as necessity to administer oxygen therapy), a multivariable logistic regression model was created. The variables for the model (age, sex, the highest d-dimer value during hospitalization, MEWS on admission, baseline LDH concentration) were selected based on the available evidence. The model was validated using the Hosmer-Lemeshow test. This was a complete-case analysis. After application of Bonferroni correction due to multiple testing, a p-value $<0.00062$ was considered statistically significant. Statistical analysis was performed with STATISTICA software (Statsoft, Tulsa, USA).

\section{Results}

\section{Baseline characteristics}

The study group consisted of 100 patients with the mean age of 59.2 (SD 16.1) years among whom 63 (63.0\%) were male. The most common comorbidities were hypertension (49.0\%), obesity (31.0\%) and dyslipidemia (19.0\%). Twenty-seven patients $(27.0 \%)$ had history of smoking. The most commonly used medications were $\beta$-blockers (35.0\%), diuretics (24.0\%), ACE inhibitors and statins (both 19.0\%). Detailed demographic and clinical characteristics are presented in Table 1.

\section{Signs and symptoms}

The most common symptoms described by the patients on admission were fever $(73.0 \%)$, cough (67.0\%) and dyspnea (44.0\%). Additionally, diarrhea and dysgeusia or dysosmia were reported by $29(29.0 \%)$ and 15 (15.0\%) patients, respectively. The median time from the onset of symptoms 
Table 1. Baseline characteristics

\begin{tabular}{|c|c|}
\hline Characteristic & Total $(n=100)$ \\
\hline \multicolumn{2}{|l|}{ Demographic } \\
\hline Age [years], mean (SD) & $59.2(16.1)$ \\
\hline Sex, male & $63(63.0 \%)$ \\
\hline Smoking & $27(27.0 \%)$ \\
\hline \multicolumn{2}{|l|}{ Symptoms } \\
\hline Time from first symptoms to positive swab [days] & $5.0(1.0-7.0)$ \\
\hline Time from first symptoms to admission [days] & $7.0(3.0-9.0)$ \\
\hline Fever prior to admission & $73(73.0 \%)$ \\
\hline Sore throat & $16(16.0 \%)$ \\
\hline Dyspnea & $44(44.0 \%)$ \\
\hline Pleuritic chest pain & $13(13.0 \%)$ \\
\hline Ischemic chest pain & $0(0.0 \%)$ \\
\hline Hemoptysis & $1(1.0 \%)$ \\
\hline Cough & $67(67.0 \%)$ \\
\hline Myalgia & $27(27.0 \%)$ \\
\hline Syncope & $2(2.0 \%)$ \\
\hline Diarrhea & $29(29.0 \%)$ \\
\hline Dysgeusia or dysosmia & $15(15.0 \%)$ \\
\hline \multicolumn{2}{|l|}{ Measurements \& vital signs on admission } \\
\hline Resting baseline $\mathrm{SpO}^{2}$ on admission [\%] & $95.0(93.0-96.0)$ \\
\hline $\mathrm{BMI}\left[\mathrm{kg} / \mathrm{m}^{2}\right]$ & $27.7(25.2-30.8)$ \\
\hline Systolic blood pressure on admission [mm Hg] & $130.0(123.5-145.5)$ \\
\hline Diastolic blood pressure on admission [mm Hg] & $82.5(77.0-90.0)$ \\
\hline Heart rate on admission [beats/minute] & $86.5(77.0-95.5)$ \\
\hline Respiratory rate on admission [breaths/minute] & $16.0(14.0-20.0)$ \\
\hline Fever on admission & $3(3.0 \%)$ \\
\hline MEWS on admission & $1.0(0.0-2.0)$ \\
\hline \multicolumn{2}{|l|}{ Comorbidities } \\
\hline Hypertension & $49(49.0 \%)$ \\
\hline Atrial fibrillation & $9(9.0 \%)$ \\
\hline Chronic heart failure & $11(11.0 \%)$ \\
\hline Coronary artery disease & $15(15.0 \%)$ \\
\hline History of myocardial infarction & $9(9.0 \%)$ \\
\hline History of stroke & $3(3.0 \%)$ \\
\hline Peripheral artery disease & $3(3.0 \%)$ \\
\hline Dyslipidemia & $19(19.0 \%)$ \\
\hline Hyperthyroidism & $0(0.0 \%)$ \\
\hline Hypothyroidism & $9(9.0 \%)$ \\
\hline Diabetes mellitus & $16(16.0 \%)$ \\
\hline Obesity & $31(31.0 \%)$ \\
\hline Chronic kidney disease & $6(6.0 \%)$ \\
\hline Asthma & $10(10.0 \%)$ \\
\hline COPD & $7(7.0 \%)$ \\
\hline
\end{tabular}




\section{Table 1. cont. Baseline characteristics}

\begin{tabular}{lc}
\hline Active neoplastic disease & $6(6.0 \%)$ \\
History of DVT/PE & $7(7.0 \%)$ \\
\hline Medications & $14(14.0 \%)$ \\
\hline Acetylsalicylic acid & $3(3.0 \%)$ \\
Oral anticoagulants & $4(4.0 \%)$ \\
Direct oral anticoagulants & $35(35.0 \%)$ \\
$\beta$-blockers & $19(19.0 \%)$ \\
ACE-I & $16(16.0 \%)$ \\
ARB & $15(15.0 \%)$ \\
Calcium channel blockers & $19(19.0 \%)$ \\
Statins & $24(24.0 \%)$ \\
Diuretics & $5(5.0 \%)$ \\
Insulin & \\
\hline
\end{tabular}

Data is presented as $\mathrm{n}$ [\%] for categorical variables and median (interquartile range) unless otherwise specified. ACE-I — angiotensin converting enzyme inhibitors; ARB — angiotensin receptor blockers; BMI — body mass index; COPD — chronic obstructive pulmonary disease; DVT/PE — deep vein thrombosis/pulmonary embolism; MEWS — modified early warning score; SD — standard deviation

to admission to the hospital was 7.0 (IQR 3.0-9.0) days. Hypotension (defined as systolic blood pressure $<90 \mathrm{~mm} \mathrm{Hg}$ ), tachycardia (defined as heart rate $>100 / \mathrm{min}$ ) and tachypnea (defined as respiratory rate $>20$ breaths/min) were observed on admission in $0(0.0 \%), 11(11.0 \%)$ and $23(23.0 \%)$ patients, respectively. The median MEWS on admission was 1.0 (IQR 0.0-2.0) point.

\section{Laboratory results}

Complete blood count most often revealed lymphopenia (35/100, 35.0\%), while thrombocytopenia was present in 17 patients $(17.0 \%)$. Coagulation tests commonly showed an increased concentration of fibrinogen (50/59, 84.7\%), d-dimer (53/84, 63.1\%) and prolonged APTT (16/89, $18.3 \%)$. Renal function, reflected by estimated glomerular filtration rate (eGFR), was normal in the majority of patients $(82 / 100,82.0 \%)$. Serum concentrations of liver enzymes were commonly elevated, i.e. alanine aminotransferase (ALT; $45 / 100,45.0 \%$ ), aspartate aminotransferase (AST; $48 / 91,52.7 \%$ ) and gamma-glutamyl transpeptidase (GGTP; 53/74, 71.6\%). The majority of patients had elevated level of lactate dehydrogenase (LDH; 78/96, 81.3\%). Among markers of inflammation we observed elevation of C-reactive protein (CRP), procalcitonin (PCT) and interleukin 6 (IL-6) in 79/100 (79.0\%), 29/96 (30.2\%) and 39/71 (54.9\%) patients, respectively. Medians with IQR as well as proportions of patients with abnormalities in laboratory results are summarized in Table 2.

\section{Imaging studies}

Chest X-ray was performed in 99 patients (99.0\%). There were no discernible pathological findings in 22 cases $(22.0 \%)$. Interstitial infiltrates and consolidations were described in $22(22.0 \%)$ and 19 patients (19.0\%), respectively. The coexistence of interstitial infiltrates and consolidations was reported in 35 patients (35.0\%). One subject had extensive neoplastic changes, which made chest X-ray analysis impossible.

Computed tomography was performed in 45 patients $(45.0 \%)$ - computed tomography pulmonary angiogram in 43 cases and high-resolution computed tomography in 2 cases. Among patients with available CT results, ground-glass opacities were described in 38 cases $(84.4 \%)$, consolidations were found in 29 patients (64.4\%), and pleural fluid was detected in 11 individuals $(24.4 \%)$.

\section{Management}

Low-molecular-weight heparin (LMWH) was administered in 90 patients $(90.0 \%)$ with the maximal administered dose being prophylactic in 39 patients $(39.0 \%)$, intermediate in 28 subjects $(28.0 \%)$ and therapeutic in 23 patients (23.0\%). The majority of patients $(69 / 100,69.0 \%)$ received antibiotics while chloroquine and ritonavir/lopinavir were administered in 36 (36.0\%) and $2(2.0 \%)$ individuals, respectively. Oxygen therapy was used in 47 patients (47.0\%) during hospitalization, with the median maximal $\mathrm{FiO}_{2}$ accounting for 50.0\% (IQR 28.0-90.0). 
Table 2. Baseline laboratory tests results

\begin{tabular}{|c|c|c|}
\hline Parameter & Value; median (IOR) & Patients with abnormal values; $n(\%)$ \\
\hline White blood count $\left[\times 10^{3} / \mathrm{mm}^{3}\right]$ & $5.95(4.56-7.59)$ & $\begin{array}{l}\text { Leukopenia: } 19 / 100(19.0 \%) \\
\text { Leukocytosis: } 11 / 100(11.0 \%)\end{array}$ \\
\hline Neutrophils, count $\left[\times 10^{3} / \mathrm{mm}^{3}\right]$ & $3.96(2.69-5.69)$ & Neutropenia: 4/100 (4.0\%) \\
\hline Lymphocytes, count $\left[\times 10^{3} / \mathrm{mm}^{3}\right]$ & $1.12(0.88-1.50)$ & Lymphopenia: 35/100 (35.0\%) \\
\hline Hemoglobin [g/dl] & $13.8(12.7-14.8)$ & Anaemia: 18/100 (18.0\%) \\
\hline Platelets $\left[\times 10^{3} / \mathrm{mm}^{3}\right]$ & $197.5(159.5-257.0)$ & Thrombocytopenia: 17/100 (17.0\%) \\
\hline D-dimer on admission [mg/L] & $0.84(0.47-1.42)$ & Elevation: 53/84 (63.1\%) \\
\hline Highest d-dimer [mg/L] & $1.20(0.74-2.44)$ & Elevation: 67/81 (82.7\%) \\
\hline Fibrinogen [g/L] & $4.7(3.7-5.9)$ & Elevation: 50/59 (84.7\%) \\
\hline APTT [s] & $31.9(28.7-35.1)$ & Prolongation: 16/89 (18.3\%) \\
\hline INR & $0.95(0.90-1.02)$ & Elevation: 4/98 (4.1\%) \\
\hline $\mathrm{eGFR}\left[\mathrm{mL} / \mathrm{min} / 1.73 \mathrm{~m}^{2}\right]$ & $87.0(66.9-104.7)$ & Decreased: $18 / 100(18.0 \%)$ \\
\hline Urea $[\mathrm{mmol} / \mathrm{L}]$ & $5.3(3.8-6.9)$ & Elevation: 17/99 (17.2\%) \\
\hline Glucose $[\mathrm{mmol} / \mathrm{L}]$ & $5.7(5.2-7.0)$ & Elevation: 48/93 (51.8\%) \\
\hline ALT $[\mathrm{U} / \mathrm{L}]$ & $31.5(20.5-61.0)$ & Elevation: $45 / 100(45.0 \%)$ \\
\hline AST [U/L] & $37.0(27.0-56.0)$ & Elevation: 48/91 (52.7\%) \\
\hline Bilirubin $[\mu \mathrm{mol} / \mathrm{L}]$ & $7.3(5.7-9.0)$ & Elevation: $0 / 85(0.0 \%)$ \\
\hline GGTP [U/L] & $49.0(26.0-107.0)$ & Elevation: 53/74 (71.6\%) \\
\hline Myoglobin $[\mu \mathrm{g} / \mathrm{L}]$ & $72.2(42.9-118.2)$ & Elevation: $21 / 70(30.0 \%)$ \\
\hline Creatine kinase [U/L] & $114.0(64.0-189.0)$ & Elevation: $22 / 90(24.4 \%)$ \\
\hline Ferritin $[\mu \mathrm{g} / \mathrm{L}]$ & $400.0(223.0-949.0)$ & Elevation: 35/71 (49.3\%) \\
\hline Lactate dehydrogenase [U/L] & $270.0(224.5-361.0)$ & Elevation: 78/96 (81.3\%) \\
\hline NT-proBNP [pg/mL] & $186.0(69.0-1061.0)$ & Elevation: 38/71 (53.5\%) \\
\hline Troponin I [ng/L] & $5.3(2.5-14.4)$ & Elevation: 7/80 (8.8\%) \\
\hline C-reactive protein [mg/L] & $37.0(9.8-91.0)$ & Elevation: 79/100 (79.0\%) \\
\hline Procalcitonin [ng/mL] & $0.04(0.02-0.11)$ & Elevation: 29/96 (30.2\%) \\
\hline Interleukin-6 [pg/mL] & $19.1(1.5-52.6)$ & Elevation: 39/71 (54.9\%) \\
\hline
\end{tabular}

ALT — alanine transferase; APTT — activated partial thromboplastin time; AST — aspartate aminotransferase; eGFR — estimated glomerular filtration rate; GGTP — gamma-glutamyl transpeptidase; INR — international normalized ratio; NT-proBNP — N-terminal pro brain natriuretic peptide

\section{Outcomes}

Among 100 enrolled patients, 10 (10.0\%) died, $47(47.0 \%)$ developed respiratory failure, 15 (15.0\%) were transferred to the ICU, 17 (17.0\%) presented AKI, 7 (7.0\%) had sepsis, and $10(10.0 \%)$ were diagnosed with pulmonary embolism. The median time of hospitalization was 19.5 days (IQR 14.0-31.5) while median time of ICU stay accounted for 12.0 (IQR 6.0-19.0) days. The majority of patients $(52 / 100,52.0 \%)$ were discharged with positive result of nasal swab for SARS-CoV-2 and were isolated at home. The median time to viral clearance among 81 subjects with available negative result was 31.0 (IQR 20.0-37.0) days. Details concerning management and outcomes are summarized in Table 3.

\section{Factors associated with severity of the} disease - the univariable analysis

Patients with severe COVID-19 had a higher MEWS on admission and more often presented with dyspnea. Moreover, in terms of laboratory results, COVID-19 patients requiring oxygen therapy were characterized by a higher white blood cell and neutrophil count, lower lymphocyte count, higher d-dimer levels as well as higher concentrations of inflammatory markers, liver damage enzymes, LDH, myoglobin, NT-proBNP and troponin. Treatment differences between these groups included more frequent administration of antibiotics and chloroquine. Detailed information about between-group differences are summarized in Table 4. A complete comparison 
Table 3. Treatment and outcomes

\begin{tabular}{lc}
\hline Treatment & Number of patients; n [\%] \\
\hline Treatment & \\
\hline Prophylactic LMWH & $39(39.0 \%)$ \\
Intermediate dose LMWH & $28(28.0 \%)$ \\
Therapeutic dose LMWH & $23(23.0 \%)$ \\
Direct oral anticoagulants & $11(11.0 \%)$ \\
Antibiotics & $69(69.0 \%)$ \\
Chloroquine & $36(36.0 \%)$ \\
Ritonavir/lopinavir & $2(2.0 \%)$ \\
Oxygen therapy on admission & $33(33.0 \%)$ \\
FiO ${ }_{2}$ on admission & $36.0(28.0-40.0)$ \\
Oxygen therapy anytime during & $47(47.0 \%)$ \\
hospitalization & \\
Highest FiO ${ }_{2}$ during hospitalisation & $5.0(28.0-90.0 \%)$ \\
\hline Outcomes & \\
\hline Mortality & $10(10.0 \%)$ \\
Mechanical ventilation & $8(8.0 \%)$ \\
Transfer to the ICU & $15(15.0 \%)$ \\
Pulmonary embolism & $10(10.0 \%)$ \\
Myocardial injury & $7(7.0 \%)$ \\
Myocardial infarction & $1(1.0 \%)$ \\
Stroke & $0(0.0 \%)$ \\
Acute kidney injury & $17(17.0 \%)$ \\
Sepsis & $7(7.0 \%)$ \\
Septic shock & $6(6.0 \%)$ \\
Length of hospitalisation [days] & $19.5(14.0-31.5)$ \\
Length of ICU stay [days] & \\
\hline
\end{tabular}

of demographic, clinical, laboratory data can be found in Supplementary Tables 1-3.

\section{Factors associated with severity of the disease - the multivariable analysis}

Multivariable analysis revealed that severe course of COVID-19 is associated with increased age (OR 1.10; 95\% CI 1.01-1.15), BMI (OR 1.24; 95\% CI 1.01-1.53), MEWS on admission (OR 3.95; 95\% CI 1.48-12.0), the highest d-dimer value during hospitalization (OR 1.73; 95\% CI 1.032.90) and baseline LDH concentration (OR 1.16; 95\% CI 1.03-1.30). The validation of the model was performed using the Hosmer and Lemeshow goodness of fit test $\left(\mathrm{X}^{2}=18.12, \mathrm{p}=0.02\right)$. Logistic regression results are summarized in Figure 1 and Supplementary Table 4.

\section{Discussion}

To our knowledge, this is one of the first manuscripts describing a prospective cohort of Polish COVID-19 patients, and we believe that it may be useful for local clinicians involved in the care of patients with SARS-CoV-2.

The presented study cohort consisted predominantly of males and the mean age amounted to 59.2 years, which confirms the results of several previous studies reporting higher representation of males in hospitalized patients [1, 2, 11, 12]. Elderly persons with COVID-19 are characterized by a high case fatality ratio and symptomatic infection rate $[13,14]$. In a Chinese modeling study, the rate of hospitalization due to COVID-19 increased with age, ranging from $1 \%$ for patients aged 20 to 29 years, through $8 \%$ for those aged 50 to 59 years, up to $18 \%$ for individuals older than 80 years [15]. Underlying medical comorbidities are considered important risk factors for severe COVID-19 course and mortality. The most common comorbidities in the presented cohort were hypertension (49\%), obesity (31\%) and dyslipidemia (19\%), which is comparable to several large studies from the United States and China. Upon initial presentation, COVID-19 symptoms are typically consistent with pneumonia [3, 16-18]. The majority of patients in this case series were hospitalized due to a cluster of flu-like symptoms, i.e. fever (73.0\%), cough (67.0\%) and dyspnea (44.0\%). Additionally, we observed a significant representation of gastrointestinal symptoms as well as other typical COVID-19 symptoms, i.e. loss of smell or taste, which was present in 15\% of cases. In a report published by the Centers for Disease Control and Prevention, the described symptoms of COVID-19 included: cough (50\%), fever $>38^{\circ} \mathrm{C}(43 \%)$, myalgia $(36 \%)$, headache (34\%), dyspnea (29\%), sore throat (20\%), diarrhea (19\%), nausea/vomiting (12\%), loss of smell or taste, abdominal pain, and rhinorrhea in fewer than 10 percent each [19].

A number of observational studies involving COVID-19 patients revealed a unique pattern of laboratory changes, encompassing hemostatic derangements and pronounced hyperinflammatory state. First, there are numerous reports about significant coagulation abnormalities, including increased concentrations of fibrinogen and d-dimer as well as relatively common prolongation 


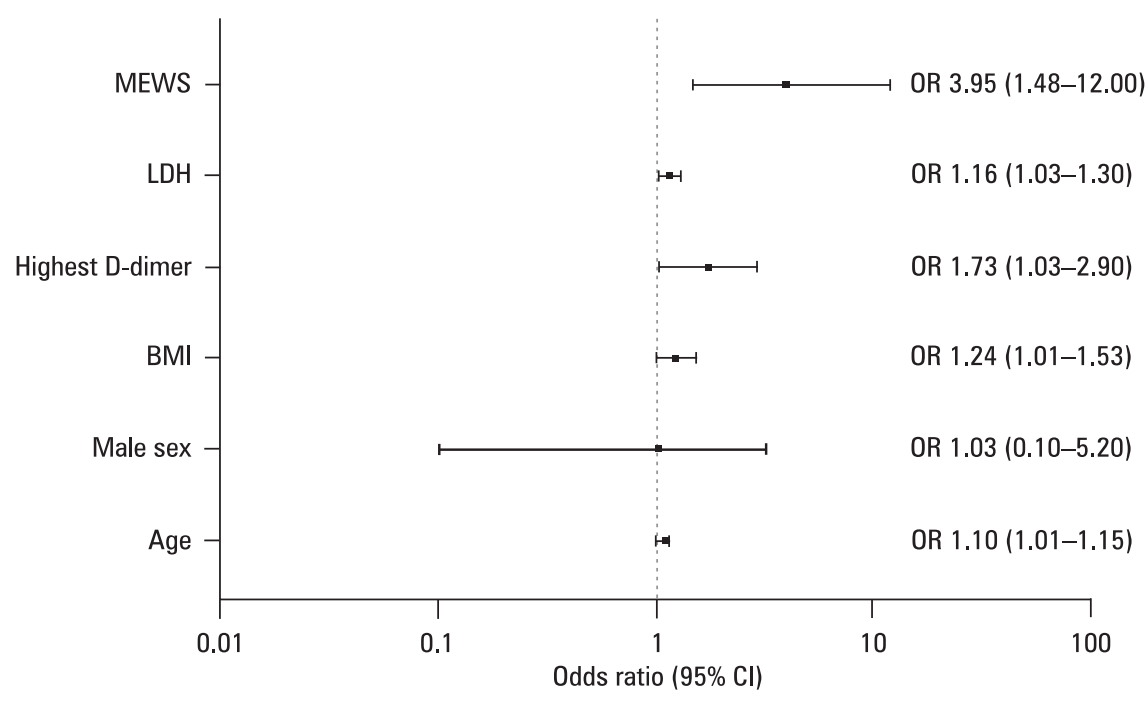

Figure 1. Summary of multivariable regression results. Dots and whiskers represent odds ratios with $95 \%$ confidence intervals. BMI — body mass index; LDH — lactate dehydrogenase; MEWS — modified early warning score; OR — odds ratio

of coagulation times. Coagulation tests in the presented cohort showed an increased concentration of fibrinogen and d-dimer in the majority of patients and prolonged APTT in nearly $20 \%$ of cases. Another frequently described laboratory anomaly is thrombocytopenia, which was present in $17 \%$ of the study group and in 5.0 to $41.7 \%$ of patients in the previous reports. According to the studies, utilizing global coagulation tests (rotational thromboelastometry, thrombography) COVID-19-associated coagulopathy represents severe hypercoagulability, which most probably is consistent neither with disseminated intravascular coagulation nor consumptive coagulopathy. Importantly, both abnormalities in coagulation tests and thrombocytopenia seem to be associated with disease severity and mortality [20]. Second, the majority of patients in the current study had laboratory findings suggestive of an exuberant inflammatory response reflected by markedly elevated concentrations of CRP, PCT, ferritin and IL-6. These observations are in line with some previous reports suggesting significant role of hyperinflammatory state with cytokine release syndrome in the pathogenesis of COVID-19 and its association with critical and fatal illness $[1,21]$. Finally, recent data suggests that liver injury is quite common among patients with COVID-19 [22]. This was also observed in the current study cohort - serum concentrations of liver enzymes (ALT, AST, GGTP) and cellular damage markers (LDH) were frequently elevated. Importantly, according to the recent meta-analysis, liver injury seems to be more prevalent in severe cases of COVID-19 [23].
Radiological findings may vary depending on disease stage, patients age, immunity status and comorbidities [24] . Normal chest radiographs or CT are found in only $18 \%$ of patients with mild disease and this proportion drops to $3 \%$ in severely ill patients. Typical CT findings in COVID-19 include bilateral, multilobar ground glass opacities with a peripheral or posterior distribution, mainly in the lower lobes, while consolidation at the initial imaging are less common (more frequent in elderly people). Moreover, pleural effusion is an uncommon but a possible finding, sometimes accompanying the disease progression [25]. In the presented study, chest radiographs were performed in nearly all patients upon admission to the hospital and similarly to data presented above, were normal in $23.9 \%$ of cases, while the remaining patients presented interstitial infiltrates, consolidations or both. Important strength of this study is high availability of CT scans which were performed in almost half of the studied patients and most commonly showed ground glass opacities followed by consolidations and presence of fluid in the pleural space.

The frequency of reported complications in COVID-19 is strongly dependent on the studied population and disease severity. The most commonly observed organ involvement in patients with severe COVID-19 is lung injury manifested by acute respiratory failure. In this study, almost half of the studied cohort developed respiratory insufficiency, and $15 \%$ of patients were transferred to the ICU due to severe respiratory failure among whom more than a half required endotra- 
Table 4. Comparison of selected variables between patients wit severe and non-severe COVID-19

\begin{tabular}{|c|c|c|c|}
\hline Characteristic & Severe cases $(n=47)$ & Non-severe cases $(n=53)$ & P-value \\
\hline \multicolumn{4}{|l|}{ Demographic } \\
\hline Age [years], mean (SD) & $62.3(15.9)$ & $56.5(15.8)$ & 0.07 \\
\hline Sex, male & $30(63.8 \%)$ & $33(62.3 \%)$ & 0.87 \\
\hline \multicolumn{4}{|l|}{ Symptoms } \\
\hline Dyspnea & $32(68.1 \%)$ & $12(22.6 \%)$ & $<0.0001$ \\
\hline \multicolumn{4}{|c|}{ Measurements and vital signs on admission } \\
\hline BMI $\left[\mathrm{kg} / \mathrm{m}^{2}\right]$ & $28.4(26.1-32.1)$ & $26.8(24.9-28.6)$ & 0.009 \\
\hline Heart rate on admission & $90.0(80.0-100.0)$ & $82.0(71.0-91.0)$ & 0.007 \\
\hline Respiratory rate on admission & $20.0(17.0-22.0)$ & $15.0(14.0-16.0)$ & $<0.0001$ \\
\hline MEWS on admission & $2.0(1.0-2.0)$ & $0.0(0.0-1.0)$ & $<0.0001$ \\
\hline \multicolumn{4}{|l|}{ Comorbidities } \\
\hline Hypertension & $29(61.7 \%)$ & $20(38.5 \%)$ & 0.02 \\
\hline Chronic heart failure & $10(21.3 \%)$ & $1(1.9 \%)$ & 0.003 \\
\hline \multicolumn{4}{|l|}{ Laboratory results } \\
\hline White blood count $\left[\times 10^{3} / \mathrm{mm}^{3}\right]$ & $6.86(5.70-9.83)$ & $5.20(3.87-6.58)$ & $<0.0001$ \\
\hline Neutrophils, count $\left[\times 10^{3} / \mathrm{uL}\right]$ & $5.34(3.92-8.15)$ & $3.02(2.03-4.01)$ & $<0.0001$ \\
\hline Lymphocytes, count $\left[\times 10^{3} / \mathrm{uL}\right]$ & $1.00(0.64-1.20)$ & $1.38(1.09-1.65)$ & $<0.0001$ \\
\hline D-dimer on admission [mg/L] & $1.10(0.74-2.08)$ & $0.54(0.35-1.07)$ & $<0.0001$ \\
\hline Highest d-dimer [mg/L] & $2.20(0.96-5.15)$ & $0.91(0.55-1.43)$ & $<0.0001$ \\
\hline APTT $[s]$ & $33.9(30.2-38.2)$ & $30.8(27.8-33.1)$ & 0.02 \\
\hline AST [U/L] & $46.0(35.0-68.0)$ & $31(24.0-41.5)$ & 0.0003 \\
\hline GGTP [U/L] & $72.5(31.0-174.0)$ & $40.0(23.0-59.0)$ & 0.003 \\
\hline Myoglobin $[\mu \mathrm{g} / \mathrm{L}]$ & $115.5(66.4-175.5)$ & $57.9(38.6-77.9)$ & $<0.0001$ \\
\hline Ferritin $[\mu \mathrm{g} / \mathrm{L}]$ & $681.0(379.0-1280.0)$ & $308.0(145.0-529.0)$ & 0.0004 \\
\hline Lactate dehydrogenase [U/L] & $354.0(284.0-456.0)$ & $232.0(201.0-275.0)$ & $<0.0001$ \\
\hline NT-proBNP [pg/mL] & $482.5(134.0-2084.0)$ & $93.0(35.0-321.0)$ & $<0.0001$ \\
\hline Troponin I [ng/L] & $12.7(4.3-29.4)$ & $3.7(2.5-7.8)$ & 0.0002 \\
\hline C-reactive protein $[\mathrm{mg} / \mathrm{L}]$ & $85.5(34.5-170.0)$ & $12.1(2.3-48.2)$ & $<0.0001$ \\
\hline Procalcitonin [ng/mL] & $0.10(0.04-0.20)$ & $0.02(0.02-0.04)$ & $<0.0001$ \\
\hline Interleukin-6 [pg/mL] & $29.3(19.1-78.0)$ & $1.5(1.5-21.2)$ & $<0.0001$ \\
\hline \multicolumn{4}{|l|}{ Treatment } \\
\hline Antibiotics & $45(95.7 \%)$ & $24(45.3 \%)$ & $<0.0001$ \\
\hline Chloroquine & $25(53.2 \%)$ & $11(20.8 \%)$ & $<0.0001$ \\
\hline \multicolumn{4}{|l|}{ Outcomes } \\
\hline Pulmonary embolism & $8(17.0 \%)$ & $2(3.8 \%)$ & 0.04 \\
\hline Acute kidney injury & $13(27.7 \%)$ & $4(7.6 \%)$ & 0.014 \\
\hline Positive swab at discharge & $19(42.2 \%)$ & $33(62.3 \%)$ & 0.047 \\
\hline
\end{tabular}


cheal intubation and mechanical ventilation. In the previous studies from the USA, $12-24 \%$ of hospitalized patients required mechanical ventilation [3, 17]. In a nationwide cross-sectional study performed in China including approximately 44,500 cases, one in twenty patients was categorized as critically ill (i.e. with respiratory failure, shock, or multiorgan dysfunction) [6]. Importantly, thromboembolic complications are markers of severe COVID-19 and are associated with multiorgan failure and increased mortality [26]. The evidence to date supports the concept that the thrombotic manifestations of severe COVID-19 are due to the ability of SARS-CoV-2 to invade endothelial cells via ACE-2 (angiotensin-converting enzyme 2). Ten percent of patients in our cohort were diagnosed with pulmonary embolism despite the fact that LMWH was administered in $90 \%$ of the analyzed cases. Depending on the population and type of study (retrospective $v s$ prospective with active screening for venous thromboembolism), the incidence of thromboembolic complications ranges from $3.9 \%$ to $79.4 \%$ in the ICU patients and from 1.3 to $14.7 \%$ among non-critically ill patients [27]. Kidney involvement in COVID-19 is frequent, with clinical presentation ranging from mild proteinuria to AKI necessitating renal replacement therapy [28]. In our study, a baseline renal function was normal in the majority of patients (82\%). In the course of the hospitalization, $7 \%$ of our patients have developed AKI, which corroborates the results of the recently published meta-analysis estimating the incidence of AKI among hospitalized patients at the level of $8.9 \%$ [29]. It is important to note that a substantial proportion of AKI could have been missed due to limited sensitivity of serum creatinine. The role of secondary infections in COVID-19 may be overestimated, although data is limited [30, 31]. In a review, the rate of bacterial or fungal coinfections was only $8 \%$, whereas in our study, $7 \%$ of hospitalized patients developed sepsis or septic shock [30].

Finding an effective treatment for COVID-19 was a long and tortuous road filled with many hopes and even more disappointments. The recruitment to our study was initiated at the beginning of pandemic in Poland, therefore, many patients received medications which are currently known to be ineffective. Chloroquine was administered to $36 \%$ of our patients while the combined protease inhibitor ritonavir/lopinavir was used extremely rarely ( $2 \%$ of cases). Unfortunately, the gradually emerging data from controlled trials revealed that these drugs do not improve outcomes in this population [32, 33]. The available evidence shows a benefit associated with the administration of systemic glucocorticoids among patients requiring oxygen therapy. The use of remdesivir is currently suggested in patients with severe COVID-19 who are not critically ill. Despite initial positive signals from observational studies, the use of convalescent plasma proved to be ineffective. Routine use of IL-6 pathway inhibitors (tocilizumab, sarilumab) is not recommended, although these drugs given in the intensive care settings may benefit a selected group of critically ill patients [34].

The mortality rate in our cohort was $10 \%$ compared to estimated mortality rate of $2.8 \%$ among the entire population of Polish COVID-19 patients [35]. The estimated mortality rate among hospitalized patients accounts for $18.9 \%$ and varies greatly depending on a study, ranging from 0.7 to $61.5 \%$ [36]. Such variability in the death rate is probably secondary to inter-country differences in population characteristics, healthcare-related factors as well as strategy concerning hospital admissions or outpatient treatment of COVID-19 patients.

Almost half the patients in this study were classified as severe cases based on the development of respiratory failure. Univariable analysis comparing severe and non-severe cases showed that patients with severe COVID-19 more commonly presented with dyspnea and increased baseline respiratory rate as well as higher MEWS on admission, thus suggesting a potential clinical utility of the latter as a risk stratification tool in this population [37]. Analysis of laboratory results revealed that severely ill patients had indicators of coagulopathy, hyperinflammatory state, liver damage and increased cardiac biomarkers [1, 16, 38].

Finding risk factors of severe COVID-19 is particularly relevant for clinician involved in management of COVID-19 patients. There are several papers reporting factors potentially associated with mortality of severe course of the disease, however, none of them is based on a Polish cohort [17, 39]. In a study of 5,279 people with COVID-19 in New York City by Petrilli et al. [17], among hospitalized patients, factors associated with critical illness were: age, heart failure, BMI (greater than 40) and male sex, with diabetes being also significant. On the other hand, in a large Chinese study, a multivariable logistic regression model showed 10 potential predictors of critical illness. These variables included chest X-ray abnormalities, age, hemoptysis, dyspnea, unconsciousness, the number of comorbidities, cancer 
history, neutrophil-to-lymphocyte ratio, as well as concentrations of LDH and direct bilirubin [39]. A multivariable analysis in our study revealed rather similar results and suggested the increasing age, BMI, MEWS on admission, the highest d-dimer level during hospitalization and LDH concentration as factors potentially related to a severe course of COVID-19. Unfortunately, a relatively small study sample reduces the statistical power of this analysis while single-center character of this study may limit its generalizability.

There is very limited data concerning the clinical characteristics and outcomes of Polish patients hospitalized due to COVID-19. In a retrospective study by Nowak et al. [40], the authors reported similar distribution of signs and symptoms as well as comorbidities in their sample. Interestingly, the mortality in our cohort was more than two times lower despite a similar proportion of critically ill patients requiring a transfer to the ICU. This may be partially due to differences in treatment, i.e. our cohort was characterized by more common administration of currently recommended LMWH as well as less frequent use of chloroquine and ritonavir/lopinavir, both of which proved to be ineffective in the treatment of COVID-19. An univariable analysis performed in the aforementioned study suggested age, shortness of breath, cardiovascular disease, malignancy and bilateral patchy shadowing in chest X-ray as potential predictors of mortality in this population, which partially corroborates our results. It is however important to note that data presented in our study was gathered in a prospective manner, and therefore, offers more valuable insight into the clinical profile of Polish patients hospitalized due to COVID-19.

This study has several limitations. First, due to the limited study sample and a relatively low incidence of several outcomes of interest, such as mortality, AKI and pulmonary embolism, we were unable to perform multivariable analysis to assess risk factors for these events. Second, this study was performed in a single ward specialized in treating patients with respiratory failure, therefore, the presented cohort may consist of patients with more severe form of COVID-19 compared to other wards, thus limiting the generalizability of the results. Third, the incidence of several outcomes might be underestimated due to lack of routine screening, i.e. lack of active troponin level monitoring for myocardial injury or lack of routine deep vein ultrasound and CT pulmonary angiogram for venous thromboembolism. Finally, we believe that this cohort of first one hundred patients with COVID-19 treated in our center comprises a relatively large proportion of mild cases compared to later stages of the pandemic.

\section{Conclusion}

In this observational study describing the clinical profile of 100 hospitalized patients with COVID-19, nearly half of the analyzed cases developed respiratory failure and approximately $10 \%$ died in the course of the disease. The multivariable analysis revealed increasing age, BMI, MEWS on admission as well as higher d-dimer and $\mathrm{LDH}$ concentration as factors associated with severe course of COVID-19.

\section{Acknowledgements}

Authors' contribution statement: All authors made substantial contributions to all of the following: 1) the concept and design of the study, or acquisition of data, or analysis and interpretation of data; 2) drafting the article or revising it critically for important intellectual content; and 3) final approval of the version to be submitted.

\section{Conflict of interest}

None declared.

\section{References:}

1. Zhang H, Du F, Cao XJ, et al. Clinical features of patients infected with 2019 novel coronavirus in Wuhan, China. Lancet. 2020; 395(10223): 497-506, doi: 10.1016/S0140-6736(20)301835, indexed in Pubmed: 31986264 .

2. Wang D, Hu Bo, Hu C, et al. Clinical characteristics of 138 hospitalized patients with 2019 novel coronavirus-infected pneumonia in Wuhan, China. JAMA. 2020; 323(11): 1061-1069, doi: 10.1001/jama.2020.1585, indexed in Pubmed: 32031570.

3. Richardson S, Hirsch JS, Narasimhan M, et al. Presenting characteristics, comorbidities, and outcomes among 5700 patients hospitalized with COVID-19 in the New York City Area. JAMA. 2020; 323(20): 2052-2059, doi: 10.1001/jama.2020.6775, indexed in Pubmed: 32320003.

4. Iba T, Levy JH, Connors JM, et al. The unique characteristics of COVID-19 coagulopathy. Crit Care. 2020; 24(1): 360, doi: 10.1186/s13054-020-03077-0, indexed in Pubmed: 32552865.

5. Fortini A, Beltrame C, Faraone A, et al. Thromboinflammatory state and venous thromboembolic events in patients with coronavirus disease 2019 admitted to a nonintensive care unit: a prospective study. Pol Arch Intern Med. 2021; 131(1): 86-89, doi: 10.20452/pamw.15625, indexed in Pubmed: 32975921.

6. Wu Z, McGoogan JM. Characteristics of and important lessons from the coronavirus disease 2019 (COVID-19) outbreak in China: summary of a report of 72314 cases from the Chinese Center for Disease Control and Prevention. JAMA. 2020; 323(13): 1239-1242, doi: 10.1001/jama.2020.2648, indexed in Pubmed: 32091533.

7. Sterne JAC, Murthy S, Diaz JV, et al. Association between administration of systemic corticosteroids and mortality among critically ill patients with COVID-19: a meta-analysis. JAMA. 2020; 324(13): 1330-1341, doi: 10.1001/jama.2020.17023, indexed in Pubmed: $\underline{32876694}$. 
8. Thygesen K, Alpert J, Jaffe A, et al. Fourth universal definition of myocardial infarction (2018). Eur Heart J. 2019; 40(3): 237-269, doi: 10.1093/eurheartj/ehy462.

9. Kellum JA, Lameire N, Aspelin P, et al. Kidney disease: Improving global outcomes (KDIGO) acute kidney injury work group. KDIGO clinical practice guideline for acute kidney injury. Kidney Int Suppl. 2012; 2(1): 1-138, doi: 10.1038/kisup.2012.1.

10. Rhodes A, Evans LE, Alhazzani W, et al. Surviving sepsis campaign: international guidelines for management of sepsis and septic shock: 2016. Crit Care Med. 2017; 45(3): 486-552, doi: 10.1097/CCM.0000000000002255, indexed in Pubmed: 28098591.

11. Liu K, Fang YY, Deng Y, et al. Clinical characteristics of novel coronavirus cases in tertiary hospitals in Hubei Province. Chin Med J (Engl). 2020; 133(9): 1025-1031, doi: 10.1097/ CM9.0000000000000744, indexed in Pubmed: 32044814.

12. Chen N, Zhou M, Dong X, et al. Epidemiological and clinical characteristics of 99 cases of 2019 novel coronavirus pneumonia in Wuhan, China: a descriptive study. Lancet. 2020; 395(10223): 507-513, doi: 10.1016/50140-6736(20)30211-7, indexed in Pubmed: 32007143.

13. Leung C. Clinical features of deaths in the novel coronavirus epidemic in China. Rev Med Virol. 2020; 30(3): e2103, doi: 10.1002/rmv.2103, indexed in Pubmed: 32175637.

14. Kang SJi, Jung SIn. Age-Related morbidity and mortality among patients with COVID-19. Infect Chemother. 2020; 52(2): 154-164, doi: 10.3947/ic.2020.52.2.154, indexed in Pubmed: 32537961.

15. Verity R, Okell LC, Dorigatti I, et al. Estimates of the severity of coronavirus disease 2019: a model-based analysis. Lancet Infect Dis. 2020; 20(6): 669-677, doi: 10.1016/S14733099(20)30243-7, indexed in Pubmed: 32240634.

16. Zhou F, Yu T, Du R, et al. Clinical course and risk factors for mortality of adult inpatients with COVID-19 in Wuhan, China: a retrospective cohort study. Lancet. 2020; 395(10229): 1054-1062, doi: 10.1016/50140-6736(20)30566-3, indexed in Pubmed: 32171076.

17. Petrilli CM, Jones SA, Yang J, et al. Factors associated with hospital admission and critical illness among 5279 people with coronavirus disease 2019 in New York City: prospective cohort study. BMJ. 2020; 369: m1966, doi: 10.1136/bmj.m1966, indexed in Pubmed: $\underline{32444366 .}$

18. Lippi G, Wong J, Henry BM. Hypertension in patients with coronavirus disease 2019 (COVID-19): a pooled analysis. Pol Arch Intern Med. 2020; 130(4): 304-309, doi: 10.20452 pamw.15272, indexed in Pubmed: 32231171.

19. Stokes EK, Zambrano LD, Anderson KN, et al. Coronavirus disease 2019 case surveillance - United States, January 22May 30, 2020. MMWR Morb Mortal Wkly Rep. 2020; 69(24): 759-765, doi: 10.15585/mmwr.mm6924e2, indexed in Pubmed: 32555134 .

20. Lippi G, Plebani M, Henry BM. Thrombocytopenia is associated with severe coronavirus disease 2019 (COVID-19) infections: A meta-analysis. Clin Chim Acta. 2020; 506: 145-148, doi: 10.1016/i.cca.2020.03.022, indexed in Pubmed: 32178975.

21. Wang C, Kang K, Gao Y, et al. Cytokine levels in the body fluids of a patient with COVID-19 and acute respiratory distress syndrome: a case report. Ann Intern Med. 2020; 173(6): 499-501, doi: 10.7326/L20-0354, indexed in Pubmed: 32422085.

22. Zhang C, Shi L, Wang FS. Liver injury in COVID-19: management and challenges. Lancet Gastroenterol Hepatol. 2020; 5(5): 428-430, doi: 10.1016/\$2468-1253(20)30057-1, indexed in Pubmed: 32145190.

23. Wong YuJ, Tan M, Zheng Q, et al. A systematic review and meta-analysis of the COVID-19 associated liver injury. Ann Hepatol. 2020; 19(6): 627-634, doi: 10.1016/j.aohep.2020.08.064. indexed in Pubmed: 32882393.

24. Rorat M, Zińczuk A, Szymański W, et al. Usefulness of a portable chest radiograph in the initial diagnosis of coronavirus disease 2019. Pol Arch Intern Med. 2020; 130(10): 906-909, doi: 10.20452/pamw.15512, indexed in Pubmed: 32666780.
25. Bao C, Liu X, Zhang H, et al. Coronavirus disease 2019 (COVID-19) CT findings: a systematic review and meta-analysis. J Am Coll Radiol. 2020; 17(6): 701-709, doi: 10.1016/i. jacr.2020.03.006, indexed in Pubmed: 32283052.

26. McFadyen JD, Stevens H, Peter K. The emerging threat of (micro)thrombosis in COVID-19 and its therapeutic implications. Circ Res. 2020; 127(4): 571-587, doi: 10.1161/CIRCRESAHA.120.317447, indexed in Pubmed: 32586214.

27. Bikdeli B, Madhavan MV, Jimenez D, et al. COVID-19 and thrombotic or thromboembolic disease: implications for prevention, antithrombotic therapy, and follow-up: JACC stateof-the-art review. J Am Coll Cardiol. 2020; 75(23): 2950-2973, doi: 10.1016/i.jacc.2020.04.031, indexed in Pubmed: 32311448.

28. Ronco C, Reis T, Husain-Syed F. Management of acute kidney injury in patients with COVID-19. Lancet Respir Med. 2020; 8(7): 738-742, doi: 10.1016/\$2213-2600(20)30229-0, indexed in Pubmed: 32416769.

29. Chen YT, Shao SC, Hsu CK, et al. Incidence of acute kidney injury in COVID-19 infection: a systematic review and meta-analysis. Crit Care. 2020; 24(1): 346, doi: 10.1186/s13054020-03009-y, indexed in Pubmed: 32546191.

30. Rawson TM, Moore LSP, Zhu N, et al. Bacterial and fungal coinfection in individuals with coronavirus: a rapid review to support COVID-19 antimicrobial prescribing. Clin Infect Dis. 2020; 71(9): 2459-2468, doi: 10.1093/cid/ciaa530, indexed in Pubmed: 32358954.

31. Sepulveda J, Westblade LF, Whittier S, et al. Bacteremia and blood culture utilization during COVID-19 surge in New York City. J Clin Microbiol. 2020; 58(8), doi: 10.1128/JCM.00875-20, indexed in Pubmed: 32404482.

32. Horby P, Mafham M, Linsell L, et al. Effect of hydroxychloroquine in hospitalized patients with COVID-19. N Engl Med. 2020; 383(21): 2030-2040, doi: 10.1056/NEJMoa2022926, indexed in Pubmed: 33031652.

33. Horby P, Mafham M, Bell J, et al. Lopinavir-ritonavir in patients admitted to hospital with COVID-19 (RECOVERY): a randomised, controlled, open-label, platform trial. The Lancet. 2020; 396(10259): 1345-1352, doi: 10.1016/s01406736(20)32013-4.

34. Alhazzani W, Evans L, Alshamsi F, et al. Surviving sepsis campaign guidelines on the management of adults with coronavirus disease 2019 (COVID-19) in the ICU: first update. Crit Care Med. 2021; 49(3): e219-e234, doi: 10.1097/ CCM.0000000000004899, indexed in Pubmed: 33555780.

35. Mortality Analyses - Johns Hopkins Coronavirus Resource Center. Available online: https://coronavirus.jhu.edu/data/mortality. [Last accessed at: October 2, 2020].

36. Noor FM, Islam MdM. Prevalence and associated risk factors of mortality among COVID-19 patients: a meta-analysis. J Community Health. 2020; 45(6): 1270-1282, doi: 10.1007/s10900020-00920-x, indexed in Pubmed: 32918645.

37. Azoulay É, Beloucif S, Guidet B, et al. Admission decisions to intensive care units in the context of the major COVID-19 outbreak: local guidance from the COVID-19 Paris-region area. Crit Care. 2020; 24(1): 293, doi: 10.1186/s13054-020-03021-2, indexed in Pubmed: 32503593.

38. Zheng Z, Peng F, Xu B, et al. Risk factors of critical \& mortal COVID-19 cases: A systematic literature review and meta-analysis. J Infect. 2020; 81(2): e16-e25, doi: 10.1016/i. jinf.2020.04.021, indexed in Pubmed: 32335169.

39. Liang W, Liang H, Ou L, et al. Development and validation of a clinical risk score to predict the occurrence of critical illness in hospitalized patients with COVID-19. JAMA Intern Med. 2020; 180(8): 1081-1089, doi: 10.1001/jamainternmed.2020.2033, indexed in Pubmed: 32396163.

40. Nowak B, Szymański P, Pańkowski I, et al. Clinical characteristics and short-term outcomes of patients with coronavirus disease 2019: a retrospective single-center experience of a designated hospital in Poland. Pol Arch Intern Med. 2020; 130(5): 407-411, doi: 10.20452/pamw.15361, indexed in Pubmed: $\underline{32420710}$. 